\title{
A Patient With a Progressive Cecal Subepithelial Tumor
}

\author{
Younjoo Kim \\ Division of Gastroenterology, Department of Internal Medicine, Korea Cancer Center Hospital, Seoul, Korea
}
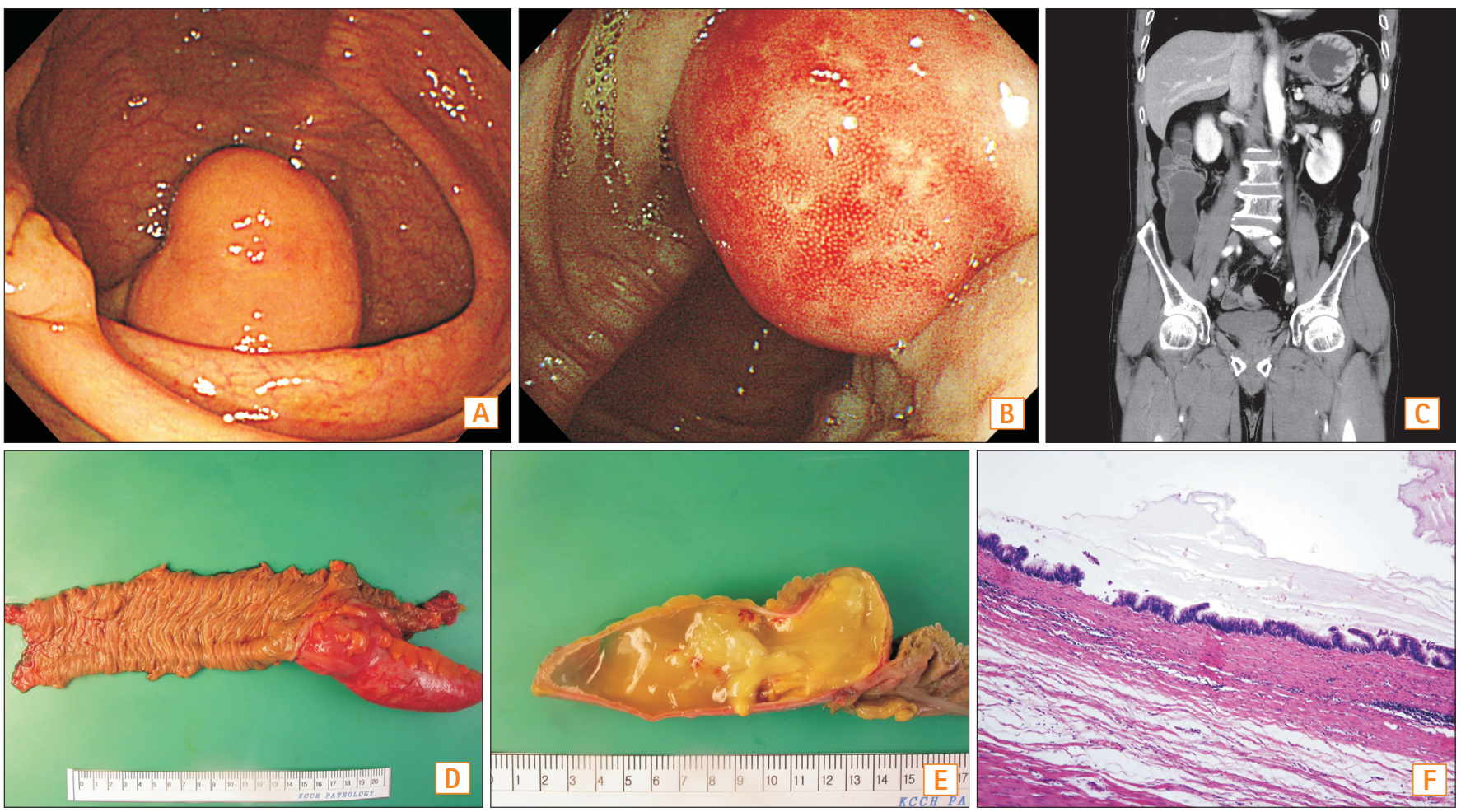

Question: A 68-year-old man visited our hospital complaining of mild discomfort in the right lower quadrant (RLQ) of his abdomen for 1 month. Three year previously, as part of a routine checkup, he had undergone a colonoscopy that had revealed a $3-\mathrm{cm}$ soft and fixed subepithelial tumor (SET) (Fig. A) However, he refused further evaluation and did not return for a follow-up visit. He reported no weight loss, but had a familial history of rectal cancer on the pater- nal side. On physical examination, his abdomen was flat and soft, with no focal tenderness or palpable masses. His hemoglobin level was $13.9 \mathrm{~g} / \mathrm{dL}$, white blood cell count was 5,510/ $\mathrm{mm}^{3}$, platelet count was $154,000 / \mathrm{mm}^{3}$, and CRP level was normal. His carcinoembryonic antigen level was $8.81 \mathrm{ng} / \mathrm{mL}$, and his carbohydrate antigen 19-9 level was $10.62 \mathrm{U} / \mathrm{mL}$.

The patient underwent colonoscopy to assess the cause of his RLQ abdominal discomfort, focusing on the previously 
detected cecal SET. A huge round SET was observed in the cecum. The mass had grown since the initial colonoscopy, and its surface color had become hyperemic. When we examined the SET with forceps, it was non-movable and had a soft consistency (Fig. B). Abdominopelvic CT revealed a 9.3-cm tubular cystic mass in the right lower abdomen (Fig. C). We recommended that the mass be resected, and laparoscopic right hemicolectomy was performed to prevent the possibility of malignancy. The resected appendix measured $12.0 \times 3.5 \times 3.5 \mathrm{~cm}$ (Fig. D). On cross-section examination, it was cystic and filled with light yellow mucinous material (Fig. E). On microscopic examination, the appendiceal wall was lined with a single layer of bland neoplastic cells resting on fibrous stroma (Fig. F; H\&E, ×100). What is the most likely diagnosis?

\section{Answer to the Images: Appendiceal Mucocele}

Appendiceal mucocele is a descriptive term indicating cystic dilatation of appendix, which is filled with mucoid material. The incidence of appendiceal mucocele has been reported to be $0.07 \%$ to $0.63 \%$, and it affects both sexes in the 5 th to 7 th decades of life. ${ }^{1,2}$ It is a heterogeneous disease rather than a single entity, and prognosis is related to histological subtypes: retention cyst, mucosal hyperplasia, mucinous cystadenoma, or mucinous cystadenocarcinoma. ${ }^{1,3}$ Nonneoplastic mucoceles are retention cysts and mucosal hyperplasia, and they are caused by simple obstruction (stricture or fecalith) or epithelial hyperplasia of the appendix. Neoplastic mucoceles are mainly composed of mucinous cystadenoma and cystadenocarcinoma. The latter is associated with pseudomyxoma peritonei. Other appendiceal malignancies include neuroendocrine tumors, non-mucinous adenocarcinomas, goblet cell carcinomas and signet ring cell carcinomas, although these rarely lead to mucocele. ${ }^{2}$ Appendiceal mucoceles are often found incidentally during colonoscopies performed for colorectal cancer screening or the evaluation of symptoms. ${ }^{1-3}$ On colonoscopy, appendiceal mucoceles are seen as extrinsic compression or SET on the cecum. The appendiceal orifice might be seen in the center of a mass (volcano sign), and mucus like material may leak out of the appendiceal orifice. ${ }^{4}$ When probed with the biopsy forceps, the lesion may be soft and exhibit a cushion sign. ${ }^{3,4}$ As the overlying mucosa is usually normal, mucosal biopsies are not sufficient to diagnose these lesions. The typical abdominal CT finding in a patient with an appendiceal mucocele is a low attenuated (similar to the filling of water), well-encapsulated round or tubular cystic mass with peripheral calcifications in the RLQ adjacent to the cecum..$^{1-3}$ Although imaging is not able to clearly distinguish between the different subtypes of mucoceles, neoplastic mucoceles tend to be larger than retention cysts. ${ }^{5}$ Additional findings of ascites, peritoneal thickening and nodules, and scalloping of the liver surface are suggestive of pseudomyxoma peritonei. On the ultrasonography including endoscopic ultrasonography, a cystic structure with echogenic multi-layers arranged in an onion skin pattern (onion skin sign) in the RLQ may be suggestive the appendiceal mucocele. ${ }^{6}$

Resection is required for these lesions, even for benignappearing appendiceal mucoceles. This is because lesions that appear to be benign on imaging studies may actually be malignant. Rupture of a neoplastic mucocele may result in the spillage of neoplastic cell contents and consequently pseudomyxoma peritonei. Therefore, careful handling and resection of these lesions is important. ${ }^{3,5}$ Standard appendectomy is suitable for retention cysts, mucosal hyperplasia, and cystadenomas. Right hemicolectomy is indicated in patients with suspicious cystadenocarcinomas. In the present case, the patient was treated with laparoscopic right hemicolectomy owing to the possibility of malignancy. Mucinous cystadenoma with clear proximal and distal resection margins was noted on the pathology report.

\section{REFERENCES}

1. Rabie ME, Al Shraim M, Al Skaini MS, et al. Mucus containing cystic lesions "mucocele" of the appendix: the unresolved issues. Int J Surg Oncol 2015;2015:139461.

2. Rymer B, Forsythe RO, Husada G. Mucocoele and mucinous tumours of the appendix: a review of the literature. Int J Surg 2015;18:132-135.

3. UpToDate ${ }^{\circledR}$ [homepage on the Internet]. Massachusetts: UpToDate, Inc.; c2015 [updated 2014 Nov 05; cited 2015 Aug]. Available from: http://www.uptodate.com/contents/appendicealmucoceles

4. Zanati SA, Martin JA, Baker JP, Streutker CJ, Marcon NE. Colonoscopic diagnosis of mucocele of the appendix. Gastrointest Endosc 2005;62:452-456.

5. Dhage-Ivatury S, Sugarbaker PH. Update on the surgical approach to mucocele of the appendix. J Am Coll Surg 2006;202: 680-684.

6. Caspi B, Cassif E, Auslender R, Herman A, Hagay Z, Appelman Z. The onion skin sign: a specific sonographic marker of appendiceal mucocele. J Ultrasound Med 2004;23:117-121. 